\title{
Insect Phylogenetics in the Digital Age
}

\author{
Christopher H. Dietrich and Dmitry A. Dmitriev
}

Illinois Natural History Survey, Prairie Research Institute, University of Illinois, $1816 \mathrm{~S}$ Oak St., Champaign, IL 61820, USA

Corresponding author: Dietrich, C. H. (chdietri@illinois.edu)

\begin{abstract}
Insect systematists have long used digital data management tools to facilitate phylogenetic research. Web-based platforms developed over the past several years support creation of comprehensive, openly accessible data repositories and analytical tools that support large-scale collaboration, accelerating efforts to document Earth's biota and reconstruct the Tree of Life. New digital tools have the potential to further enhance insect phylogenetics by providing efficient workflows for capturing and analyzing phylogenetically relevant data. Recent initiatives streamline various steps in phylogenetic studies and provide community access to supercomputing resources. In the near future, automated, web-based systems will enable researchers to complete a phylogenetic study from start to finish using resources linked together within a single portal and incorporate results into a global synthesis.
\end{abstract}

\section{Introduction}

Digital tools and resources are revolutionizing insect phylogenetics. Over the past 50 years, as computing power and storage capacity have grown and become affordable, and programming technologies have become more versatile, automation of many of the steps involved in a phylogenetic study has become possible. Tools for managing field sampling data and notes, curating and managing specimens, and compiling and analyzing phenotypic and genomic data continue to be improved and Internet-accessible databases useful for systematics research (e.g., scientific names, specimen occurrence data, DNA sequences, morphology, images) are growing exponentially.

Recent advances and grand challenges in biodiversity informatics overall have been reviewed recently [e.g., 1, 2]. This review deals more specifically with progress on digital resources relevant to insect systematics.

\section{Data and Specimen Acquisition}

Specimens are the raw materials upon which phylogenetic research is based. Thus, specimen acquisition is the crucial first step in any phylogenetic study not based on previously published data. Mass insect sampling methods (e.g., insecticidal fogging of forest canopy) adopted over the past several decades are adding vast quantities of new specimens to the billions already housed in museums, but processing and identification of specimens remains a major bottleneck and practical methods for streamlining these traditionally painstaking tasks have not yet been developed [3]. Methods for bypassing manual specimen sorting and processing using DNA barcoding and/or genome sequencing technologies on bulk samples are now routinely applied to microbial faunas 
[4] and have also been applied to macrofauna $[5,6,7,8]$ although, for insects, such efforts continue to be undertaken in parallel to more traditional morphology-based identification and vouchering of individual specimens representing particular taxa or operational taxonomic units or OTUs $[9,10,11]$.

Given the logistical hurdles (e.g., obtaining permits) and high costs involved in field sampling, once samples have been obtained, they remain invaluable for ongoing species discovery [12]. Mechanisms for preserving and disseminating partially processed bulk samples and associated data need to be improved. The Global Genome Biodiversity Network [13] is attempting to address the community need for long-term archival storage and access to genome-quality samples but has, so far, incorporated only a tiny fraction of the available specimens, representing a miniscule proportion of global biodiversity.

Capture and management of field data. When collecting new specimens in the field, biologists have traditionally recorded data on the time and place of collection of each sample and other information (e.g., ecological associations, collecting methods) in field notebooks. The existence of web-integrated biodiversity database applications [e.g,14] now makes it possible to access digital data entry forms in the field. Collection management database software (either web- or desktop-based) can be used to create a permanent digital record for each collecting event, generate specimen labels (including unique identifiers) and track the provenance and curation history of each specimen in the sample throughout the curatorial process.

Capture of legacy data from collections. Capturing data from specimens previously deposited in collections is difficult because the data first need to be digitally transcribed and interpreted, and specimens originating from a single collecting event may be dispersed over several locations in the collection. Recent initiatives (e.g., https://www.idigbio.org/) have accelerated the pace of retrospective collection digitization but, despite these efforts, data for the vast majority of specimens in most extant insect collections remain inaccessible [15]. High-throughput digitization methods including whole-drawer imaging of pinned insect collections $[16,17]$ combined with some combination of crowdsourced and or automated image segmentation and label data capture [18] may provide the most cost-effective approach to capturing data for the billions of extant specimens but these efforts have begun only recently and most insect collection digitization efforts are still using traditional specimen-level manual digitization.

Capture of morphological data. Although ongoing phylogenetic studies are incorporating increasing amounts of molecular data (Kjer et al., this volume), insect taxonomy is still largely morphology based and integrative analysis of morphological and molecular data is widely regarded as the best approach to insect systematics [19,20]. Morphological evolution also remains a major research focus and advanced digital technologies (e.g., microCT) are providing images of unprecedented detail and quality, fueling a renaissance in insect comparative morphology (Wipfler et al., this volume) including the study of extant taxa as well as fossils (Ware \& Barden, this volume). Compilation of 
morphological data has traditionally required painstaking comparative study to assess qualitative and/or quantitative differences among specimens, determine which features are potentially diagnostic and phylogenetically informative, and produce a taxoncharacter state matrix. The resulting matrix may be incorporated into a phylogenetic analysis and to generate taxonomic descriptions and identification keys. Cybertaxonomic software can dramatically increase taxonomic consistency and productivity by enabling rapid searches of character space, facilitating revisionary syntheses comprising hundreds of taxa [21].

Advances in machine vision and machine learning technologies have begun to facilitate automated capture of character data from specimen images. The feasibility of using such technology for automated insect species identification based on both traditional geometric measurements [e.g., 22] and non-traditional features (e.g., reflectance [23]) has already been demonstrated and such systems may be just as accurate, or more so, than human taxonomists. Some authors have also suggested the possibility of automating capture of morphological character data from specimens for use in phylogenetic analysis [24,25]. Although, to our knowledge this has not been done successfully, given improvements in computing technologies and expanding digital libraries comprising millions of high-resolution specimen images, it may soon be feasible to develop more comprehensive and sophisticated automated data acquisition tools.

Morphological data may also be extracted from existing taxonomic literature using standardized text markup [26], ontologies [27] and natural language processing tools [28-30]. Unfortunately, published descriptions are often highly inconsistent and imprecise in their use of terminology and descriptions of different taxa within the same group often do not consistently include the same characters in the same order. Although morphological ontologies [27] may help overcome such inconsistencies, the construction of such ontologies is, itself, time-consuming. Thus, although similar tools have been used successfully to parse text for other kinds of data relevant to phylogenetics (e.g., scientific names, geographic localities) [31] we view the potential for mining legacy taxon descriptions for useful phylogenetic data as being extremely limited and usually inferior to published morphological illustrations or specimen images as sources of data.

Tools for acquiring molecular data in insect phylogenetics are described in detail elsewhere in this volume (Kjer et al., Yeates et al., this volume).

\section{Data Management and Synthesis}

Once acquired, phylogenetically relevant data need to be managed and integrated with other kinds of data to facilitate analysis, sharing and long-term preservation. In addition to the morphological or molecular character data used in the phylogenetic analysis, taxonomic nomenclature, specimen-level occurrence data, bibliographic data and images are also integral to systematics research. Although various software is available for managing phylogenetic data, most available systems manage a few kinds of data very well but have limited or no support for integrating other kinds of data relevant to phylogenetic revisionary studies. For example, specimen-level collection management databases (e.g., Arctos [https://arctosdb.org/], Specify [http://specifyx.specifysoftware.org/], Symbiota [http://symbiota.org/docs/]) integrate 
taxonomic nomenclature with specimen-level occurrence data, provide user-friendly data entry forms and include tools for managing specimen loans and generating reports, and some incorporate georeferencing tools to facilitate retrospective capture of map coordinates. But because they were designed for collection management, such systems do not support inclusion of character data for use in phylogenetics. Other packages (e.g., Mesquite [http://mesquiteproject.org]) are used primarily for managing character state data and include analytical (including tree-building and character tracing) tools but provide limited or no support for other kinds of data relevant to systematics such as specimen-level occurrence data.

3i [21; http://dmitriev.speciesfile.org/index.asp] and $m x$

[http://mx.phenomix.org/index.php/Main Page] represent two attempts to provide more comprehensive sets of tools for managing data relevant to phylogenetic systematics. Both support incorporation of specimen-level occurrence data as well as OTU-specific character state data, images, taxonomic nomenclature, and bibliographic data. Both also include tools for automatically generating keys; $3 \mathrm{i}$ also generates natural language taxon descriptions. The creators of $3 \mathrm{i}$ and $\mathrm{mx}$ are now collaborating to replace these legacy systems with a more comprehensive, entirely web-integrated system called TaxonWorks that can both facilitate systematics research and integrate with other online tools and data portals. Other web-integrated systems already available that facilitate management of phylogenetically relevant data include Morphobank [32], which facilitates upload, collaborative development and analysis of morphological datasets and supports linking images to individual matrix cells, and MorphBank [http://www.morphbank.net/], which is focused primarily on management and annotation of morphological images.

\section{Data Analysis}

Despite the development of increasingly efficient programs for analyzing phylogenetic datasets using desktop computers, the flood of phylogenetic data resulting from inexpensive genome-sequencing technologies has begun to overwhelm the capacity of desktop computers to conduct phylogenetic analyses in reasonable amounts of time. Efforts have already been made to broaden access to supercomputing resources by the systematics community. These include the web portals Phylogeny.fr [33], T-REX [34], CIPRES [35] and W-IQ-TREE [36] that provide access to various analytical tools customizable to various degrees and including blast search, multiple sequence alignment, phylogenetic analysis using various methods, calculation of branch support, and tree visualization. Several additional websites provide access to individual tools as web services [e.g., 37,38] and these may be linked together by users to create customized analytical pipelines. Unfortunately, even these recent initiatives struggle to keep up with the growth in size of phylogenomic datasets.

\section{Data Dissemination}

The Convention on Biological Diversity [https://www.cbd.int/convention/text/] and the Nagoya Protocol on Access to Genetic Resources and the Fair and Equitable Sharing of Benefits Arising from their Utilization [http://www.cbd.int/abs/doc/protocol/nagoyaprotocol-en.pdf] established goals for sharing and open access to specimens and data 
within the global scientific community but these standards have not yet been fully implemented. Initiatives such as the Genomic Observatories initiative and the Global Genome Initiative have begun to establish infrastructure but the success of such initiatives will require broad based support by the research community. Biodiversity- and phylogeny-focused web-based platforms include: (1) global specimen-level data portals such as GBIF and iDigBio as well as smaller-scale portals targeting particular taxa and/or research communities [14,39]; (2) georeferencing tools and databases [40]; (3) morphological data/image portals (e.g., Morphbank and Morphobank); (4) literature (e.g., Biodiversity Heritage Library [http://www.biodiversitylibrary.org/], Bibliography of Life [http://plazi.org/resources/bibliography-of-life-bol/]; (5) nomenclature [31], CoL

[http://www.catalogueoflife.org/], wikiSpecies

[https://species.wikimedia.org/wiki/Main Page], Global Names Architecture

[http://globalnames.org/], ZooBank [http://zoobank.org/]); and (6) phylogeny (Tree of Life Web Project [http://tolweb.org/], Open Tree of Life [https://tree.opentreeoflife.org/]). The biodiversity informatics community, represented by Biodiversity Information Standards or TDWG (www.tdwg.org), has proposed protocols (e.g., Darwin Core) to facilitate exchange of various kinds of data among institutional databases and community portals and these continue to evolve in response to the need for increased integration of disparate data types. Publishers such as ZooKeys and the Biodiversity Data Journal are also developing innovative platforms supporting publication of non-static content such as interactive keys, 3D images, sound recordings, etc. [41].

\section{Concluding Remarks}

Technological hurdles hindering progress towards development of a comprehensive tree of life are being rapidly overcome but many barriers remain, including the social preference among many systematists for solitary research as opposed to large-scale collaboration $[42,1,2]$. Despite nearly 300 years of effort, insect systematists have, so far, only documented a small fraction $(<20 \%)$ of extant insect biodiversity, large numbers of documented species are known from only a few museum specimens with minimal occurrence data [43] and traditional specimen preservation methods are not conducive to the preservation of genomic DNA. Many species are being driven to extinction before they have even been discovered [44] and only a small fraction of known insect species have ever been incorporated into an explicit phylogenetic analysis. Thousands of institutions worldwide house specimens of potential value for phylogenetic research but, because the vast majority of these holdings have not been digitized, they remain largely inaccessible [15]. Efforts are needed to stabilize, process and disseminate recent bulk samples and obtain new specimens, with improved data on ecological associations, to expand the raw materials available for phylogenomic and evolutionary research.

Digital infrastructure is being developed for storing and manipulating the vast quantities of new genomic and phenotypic data yielded by next-generation DNA sequencing and advanced digital imaging technologies but the majority of the global systematics community, particularly in developing countries, continues to focus on alpha taxonomic research. These biodiversity scientists constitute a reserve army of experts 
whose detailed knowledge of particular taxa and/or regional faunas is currently underutilized in ongoing, large-scale initiatives to reconstruct the Tree of Life.

Availability of high-throughput methods for obtaining genome-scale data from large numbers of taxa at low cost has provided new opportunities to construct robust phylogenetic estimates for diverse insect taxa. Advanced digital workflows and supercomputing resources are also available for analyzing genome-scale datasets. Nevertheless, access to many such resources is currently limited to those with specialized expertise in bioinformatics and phylogenomics and will need to be expanded in order to meet community demand and to accelerate progress towards a comprehensive Tree of Life. Because the ranks of insect systematists are not expanding to keep pace with the ongoing biodiversity crisis, it is imperative that already practicing systematists and their students form broadly collaborative global networks and have access to the tools, resources and training that enable them to do their work more efficiently.

The major challenge remaining is to link the available tools together into comprehensive workflows that automate the process of conducting a phylogenetic revisionary study from start to finish. Given current trends, we anticipate the development of increasingly integrated, open-access, web-based systems that (1) provide access to comprehensive digital data relevant for phylogenetics, (2) incorporate the data management, analytical and visualization tools for phylogenetic research already implemented in desktop systems, (3) facilitate development of additional tools and processes that further automate and streamline research in phylogenetic systematics. The ultimate goal of such efforts is a robust, sustainable, openly-accessible research platform and data repository that integrates data acquisition, storage and analytical tools to produce a globally comprehensive Tree of Life, facilitate taxon identification and provide access to taxon- and lineage-specific information.

\section{Acknowledgments}

C.H. Dietrich and D.A. Dmitriev were funded, in part, by the U.S. National Science Foundation [grants EF-1115112, DEB-1239788, and DBI-1458285]. Any opinions, findings, and conclusions or recommendations expressed in this article are those of the authors and do not necessarily reflect the views of the National Science Foundation.

\section{Literature Cited}

1. Hardisty A, Roberts $D$, et al.: A decadal view of biodiversity informatics: challenges and priorities. BMC Ecology 2013, DOI: 1472-6785/13/16

\section{Peterson AT, Soberón J, Krishtalka L: A global perspective on decadal challenges} and priorities in biodiversity informatics. BMC ECol. 2015, DOI 10.1186/s12898-0150046-8

\section{Häuser CL, Riede K: Field methods for inventorying insects. In Descriptive} taxonomy: the foundation of biodiversity research. Edited by Watson MF, Lyal C, Pendry C. Cambridge University Press; 2015:190-213. 
4. *Cristescu M: From barcoding single individuals to metabarcoding biological communities: towards an integrative approach to the study of global biodiversity. Trend. Ecol. Evol. 2014, 29:566-571.

This is a useful review of methods and issues related to use of high-throughput DNA sequencing methods to document the components of biological communities.

5. Gibson J, Shokralla S, Porter TM, King I, van Konynenburg S, Janzen DH, Hallwachs W, Hajibabaei M: Simultaneous assessment of the macrobiome and microbiome in a bulk sample of tropical arthropods through DNA metasystematics. Proc. Nat. Acad. Sci. 2014, 111:8007-8012.

6. *Crampton-Platt A, Timmermans MJTN, Gimmel ML, Kutty SN, Cockerill TD, Khen $\mathrm{CH}$, Vogler AP: Soup to tree: the phylogeny of beetles inferred by mitochondrial metagenomics of a Bornean rainforest sample. 2015, Mol. Biol. Evol. 32:2302-2316. This is a good recent example of an attempt to use phylogenetic analysis of molecular data to streamline the process of documenting biodiversity in a poorly sampled but highly diverse insect fauna.

7. Elbrecht V, Taberlet $P$, Dejean T, Valentini A, Usseglio-Polatera $P$, Beisel J-N, Coissac $\mathrm{E}$, Boyer F, Lees F: Testing the potential of a ribosomal 16S marker for DNA metabarcoding of insects. PeerJ 2016, DOI: 10.7717/peerj.1966 8. Meier R, Wong W, Srivathsan A, Foo M: \$1 DNA barcodes for reconstructing complex phenomes and finding rare species in specimen-rich samples. Cladistics 2016, 32:100-110.

9. Pocco ME, Minutolo C, Dinghi PA, Lange CE, Confalonieri VA, Cigliano MM: Species delimitation in the Andean grasshopper genus Orotettix Ronderos \& Carbonell (Orthoptera: Melanoplinae): an integrative approach combining morphological, molecular and biogeographical data. Zool. J. Linn. Soc. 2015, 174:733-759.

10. *Santos APM, Takiya DM, Nessimian JL: Integrative taxonomy of Metrichia Ross (Trichoptera: Hydroptilidae: Ochrotrichiinae) microcaddisflies from Brazil: descriptions of twenty new species. PeerJ 2016, DOI: 10.7717/peerj.2009. This is a good example of how an integrative approach to taxonomy can improve knowledge of phylogeny and speciation as well as provide tools for identification of poorly known tropical faunas.

11. Thormann B, Ahrens D, Armijos DM, Peters MK, Wagner T, Wägele JW: Exploring the leaf beetle fauna (Coleoptera: Chrysomelidae) of an Ecuadorian mountain forest using DNA barcoding. PLOS One 2016, DOI:10.1371/journal.pone.0148268

12. Weirauch C, Frankenberg S: From "insect soup" to biodiversity discovery: taxonomic revision of Peloridinannus Wygodzinsky 1951 (Hemiptera: 
Schizopteridae), with description of six new species. Arthropod Syst. Phyl. 2015, 73:457-475.

13. Droege G, Barker K, Astrin JJ, Bartels P, Butler C, Cantrill D, Coddington J, Forest F, Gemeinholzer B, Hobern D, Mackenzie-Dodds J, et al.: The Global Genome Biodiversity Network (GGBN) Data Portal. Nucl. Ac. Res. 2013, 42: D607-D612.

14. *Gries C, Gilbert EE, Franz NM: Symbiota -- a virtual platform for creating voucher-based biodiversity information communities. Biodiv. Data J. 2014, DOI: 10.3897/BDJ.2.e1114

The Symbiota platform described in this paper has become one of the most widely used sets of integrated web tools supporting communities of collections, including several Thematic Collection Networks supported by the US National Science Foundation's Advancing Digitization of Biodiversity Collections program.

15. Blagoderov V, Kitching IJ, Livermore L, Simonsen TJ, Smith VS: No specimen left behind: industrial scale digitization of natural history collections. ZooKeys 2012, 209:133-146.

16. Dietrich CH, Hart J, Raila D, Ravaioli U, Sobh N, Sobh O, Taylor C: InvertNet: a new paradigm for digital access to invertebrate collections. ZooKeys 209:165-181.

17. Balke M, Schmidt S, Hausmann A, Toussaint E, Bergsten J, Buffington M, Häuser CL, Kroupa A, Hagedorn G, Riedel A, Polaszek A, et al.: Biodiversity in your hands - a call for a virtual global natural history "metacollection." Front. Zool. 2013, DOI:10.1186/1742-9994-10-55

18. *Hudson LN, Blagoderov V, Heaton A, Holtzhausen P, Livermore L, Price BW, van der Walt S, Smith VS: Inselect: automating the digitization of natural history collections. PLoS One 2015, DOl::10.1371/journal.pone.0143402

This paper provides a well argued justification for high-throughput digitization of natural history collections and describes innovative software for segmenting and annotating individual specimen images in whole drawer panoramas.

19. Padial J, Miralles A, De la Riva I, Vences M: The integrative future of taxonomy. Front. Zool. 2010, 7:16.

20. Riedel A, Sagata K, Suhardjono YR, Tänzler R, Balke M: Integrative taxonomy on the fast track -- towards more sustainability in biodiversity research. Front. Zool. 2013, 10:15.

21. Dmitriev DA: 3I, a new program for creating Internet-accessible interactive keys and taxonomic databases and its application for taxonomy of Cicadina (Homoptera). Russ. Entomol. J. 2006, 15:263-268. 
22. Favret C, Sieracki JM: Machine vision automated species identification scaled towards production levels. Syst. Entomol. 2016, 41:133-143.

23. Wang $Y$, Nansen $C$, Zhang $Y$ : Integrative insect taxonomy based on morphology, mitochondrial DNA, and hyperspectral reflectance profiling. Zool. J. Linn. Soc. 2016, 177:378-394.

24. LaSalle J, Wheeler Q, Jackway P, Winterton S, Hobern D, Lovell D: Accelerating taxonomic discovery through automated character extraction. Zootaxa 2009, 2217: 43-55.

25. Burleigh JG, Alphonse K, Alverson AJ, Bik HM, Blank C, Cirranello AL, Cui H, Daly M, Dietterich T, Gasparich G, et al.: Next-generation phenomics for the Tree of Life. PLoS Currents 2013, DOI: 10.1371/currents.tol.085c713acafc8711b2ff7010a4b03733

26. *Miller J, Agosti D, Penev L, Sautter G, Georgiev T, Catapano T, Patterson D, King $D$, Pereira S, Vos RA, Sierra S: Integrating and visualizing primary data from prospective and legacy taxonomic literature. Biodiv. Data J. 2015, DOI: 10.3897/BDJ.3.e5063

This paper reviews tools and methods for capturing data from taxonomic texts.

27. Yoder MJ, Miko I, Seltmann K, Bertone M, Deans AR: A gross anatomy ontology for Hymenoptera. PLoS One 2010, DOI:10.1371/journal.pone.0015991 28. Cui $\mathrm{H}$ : CharaParser for fine-grained semantic annotation of organism morphological descriptions. J. Am. Soc. Inf. Sci. Tech. 2012, 63:738-754.

29. Thessen AE, Cui $H$, Mozzherin D: Applications of natural language processing in biodiversity science. Adv. Bioinf. 2012, DOI:10.1155/2012/391574

30. *Lyal C: Digitising legacy taxonomic literature: processes, products and using the output. ZooKeys 2016, 550: 189-206.

This is a useful review of methods and efforts to parse legacy taxonomic literature to obtain data (e.g., on morphology, geographic distributions, nomenclature) useful for ongoing biodiversity studies.

31. *Patterson D, Mozzherin D, Shorthouse DP, Thessen A: Challenges with using names to link digital biodiversity information. Biodiv. Data J. 2016, DOI:10.3897/BDJ.4.e8080

This paper provides a useful overview of ongoing issues with taxonomic nomenclature and how these affect efforts to create a globally comprehensive database of scientific names. 
32. O'Leary M, Kaufman S: MorphoBank: phylophenomics in the "cloud." Cladistics 2011, 27:529-537.

33. Dereeper A, Guignon V, Blanc G, Audic S, Buffet S, Chevenet F, Dufayard JF, Guindon S, Lefort $\mathrm{V}$, Lescot $\mathrm{M}$, et al.: Phylogeny.fr: robust phylogenetic analysis for the non-specialist. Nucleic Acids Research 2008, 36:W465-469.

34. Boc A, Diallo AB, Makarenkov V: T-REX: a web server for inferring, validating and visualizing phylogenetic trees and networks. Nuc. Ac. Res. 2012, 40:W573W579.

35. *Miller M, Pfeiffer W, Schartz T: The CIPRES science gateway: enabling highimpact science for phylogenetics researchers with limited resources. Proceedings of the 1st Convergence of the Extreme Science and Discovery Environment: Bridging from the eXtreme to the campus and beyond 2012, 39.

36. Trifinopoulos J, Nguyen, L-T, von Haeseler A, Minh BQ: W-IQ-TREE: a fast online phylogenetic tool for maximum likelihood analysis. Nuc. Ac. Res. 2016, 4:W232235.

37. Goecks J, Nekrutenko A, Taylor J: Galaxy: a comprehensive approach for supporting accessible, reproducible, and transparent computational research in the life sciences. Genome Biol. 2010, 11:R86.

38. Bazinet, AL, Zwickl, DJ, Cummings, MP: A gateway for phylogenetic analysis powered by grid computing featuring GARLI 2.0. Syst. Biol. 2014, 63:812-818.

39. Smith V, Rycroft S, Brake I, Scott B, Baker E, Livermore L, Blagoderov V, Roberts D: Scratchpads 2.0: a virtual research environment supporting scholarly collaboration, communication and data publication in biodiversity science. ZooKeys 2011, 150:53-70.

40. *van Erp M, Hensel R, Ceolin D, van der Meij M: Georeferencing animal specimen datasets. Trans. GIS 2015, 19: 563-581.

This paper provides a thorough review of problems and tools related to retrospective georeferencing of specimen occurrence records from collections.

41. Stoev P, Penev L, Akkari N, Cheung DK, Enghoff H, Brunke A, de Souza CM, Pape $T$, Mietchen $D$, Erwin $T$ : Revolving images and multi-image keys open new horizons in descriptive taxonomy: ZooKeys working examples. ZooKeys 2013, 328:1-3.

42. Johnson NF: A collaborative, integrated and electronic future for taxonomy. Invert. Syst. 2011, 25:471-475. 
43. Staab M, Ohl M, Zhu C-D, Klein A-M: Observational natural history and morphological taxonomy are indispensable for future challenges in biodiversity and conservation. Comm. Integr. Biol. 2015, 8:1.

44. Régnier C, Achaz G, Lambert A, Cowie RH, Bouchet $P$, Fontaine B: Mass extinction in poorly known taxa. Proc. Nat. Acad. Sci. 2015, 112:7761-7766.

This paper highlights the existence of a largely hidden biodiversity crisis among poorly studied invertebrate taxa, accentuating the need for more efficient and collaborative workflows to document global biodiversity. 